\title{
АНАЛИЗ СОМАТОГЕННОГО ВЛИЯНИЯ ГИПЕРВЕНТИЛЯЦИОННОГО СИНДРОМА В АЛЬТЕРНАТИВНЫХ ГРУППАХ ОБСЛЕДУЕМЫХ, ДИФФЕРЕНЦИРОВАННЫХ ПО КАРДИОЛОГИЧЕСКОМУ СТАТУСУ
}

\section{THE ANALYSIS \\ OF THE HYPERVENTILATION SYNDROME SOMATOGENIC IMPACT IN ALTERNATIVE SURVEY-GROUPS, WHICH WERE DIFFERENTIATED BY CARDIOLOGICAL STATUS}

N. Voronin

S. Kuznetsov

D. Kuznetsov

Summary. The article presents data from an original study to assess the actual frequency of occurrence and significance in the formation of the severity of the somatic state of the body in three groups of subjects with different cardiac status - conventionally healthy young people, elderly patients with chronic heart failure and patients with unstable angina pectoris occurring against the background of type 2 diabetes mellitus. It was found that in all three groups the actual incidence of hyperventilation syndrome is higher than it was previously thought at the level of traditional ideas. In addition, its presence has somatic significance for subjects with any cardiac status, and it increases as the severity of involvement in the pathological process of the cardiovascular system increases. For conventionally healthy young people, it causes subclinical negative consequences, for patients with chronic heart failure moderate clinically significant negative consequences, for patients with unstable angina pectoris associated with type 2 diabetes mellitus pronounced clinical negative consequences in the form of worsening of the course and outcomes of the disease. The results obtained are distinguished by their novelty and practical significance, which makes it possible to recommend them for further consideration in the practice of specialists in the clinic of internal diseases.

Keywords: hyperventilation syndrome (HVS), conditionally healthy, chronic heart failure (CHF), unstable stenocardia, diabetes mellitus, somatic significance.

\section{Введение}

$\Phi$ актические и потенциальные факторы возникновения и прогрессии кардиальной патологии продолжают представлять значительный научно-практический интерес $[13,16,17]$. Одним из них является т.н. «гипервентиляционный синдром» (ГВС) $[1,19]$, действительное значение которого продолжительное
Воронин Никита Игоревич

Д.м.и., ФГБОУ ВО «Тамбовский государственный университет имени Г.Р. Державина» nikita-tmb@yandex.ru

Кузнецов Сергей Иванович

Д.м.н., профессор, Воронежский Государственный Медицинский Университет имени Н.Н. Бурденко, врачкардиолог, г. Воронеж, 09ksi@mail.ru

Кузнецов Дмитрий Сергеевич Врач-кардиолог, БУз ВО «Воронежская областная клиническая больница № 1», г. Воронеж

Аннотация. В статье представлены данные оригинального исследования по оценке фактической частоты встречаемости и значимости в формировании тяжести соматического состояния организма у трех групп обследуемых с различным кардиологическим статусом - условно здоровых лиц молодого возраста, пациентов пожилого возраста с хронической сердечной недостаточностью и пациентов с нестабильной стенокардией, протекающей на фоне сахарного диабета второго типа. Установлено, что во всех трех группах фактическая встречаемость гипервентиляционного синдрома является выше таковой, чем это считалось ранее на уровне традиционных представлений. Помимо этого, его присутствие имеет соматическую значимость для обследуемых с любым кардиологическим статусом, причем она возрастает по мере увеличения тяжести вовлечения в патологический процесс сердечно-сосудистой системы. Для условно здоровых лиц молодого возраста он вызывает субклинические негативные последствия, для пациентов с хронической сердечной недостаточностью - умеренные клинически значимые негативные последствия, для пациентов с нестабильной стенокардией на фоне сахарного диабета второго типа — выраженные клинические негативные последствия в виде ухудшения течения и исходов заболевания. Полученные результаты отличает новизна и практическая значимость, что позволяет рекомендовать их к дальнейшему учету в практике специалистов клиники внутренних болезней.

Ключевые слова: гипервентиляционный синдром (ГВС), условно здоровые, хроническая сердечная недостаточность (ХСН), нестабильная стенокардия, сахарный диабет, соматическая значимость.

время было недооценено, однако на современном этапе внимание клиницистов к нему неуклонно возрастает $[2,4,5,14,18,20]$. В этом смысле существенное значение имеет перманентный анализ его экспрессии (в аспекте влияния на тяжесть общесоматического состояния) у альтернативных друг другу групп обследуемых, $\partial u ф$ ференцированных в зависимости от исходного кардиологического статуса. В данном отношении для по- 
добных оценок в роли наиболее «легкого» контингента из всех возможных могут выступать условно здоровые лица молодого возраста $[11,12,13,15]$, в качестве «среднетяжелого» - пациенты пожилого возраста с хронической сердечной недостаточностью (ХСН) [Малая, 2007], в качестве наиболее тяжелого - пациенты с нестабильной стенокардией [17], в особенности - осложненной коморбидными дисметаболическими состояниями $[3,9,10]$, среди которых сахарный диабет второго типа занимает одно из ведущих мест [3,6]. Однако, несмотря на целесообразность проведения подобных уточняющих исследований, до настоящего времени они практически не реализованы.

\section{Теоретический анализ}

Исследования, направленные на изучение вклада в формирование общесоматического состояния организма кардиальной патологии $[10,16,17]$ и гипервентиляционного синдрома $[1,2,4,5,11,14,19,20]$ достаточно продолжительно проводятся как в России $[1,2,4,7,8,9,10,11.14,16,17]$, так и за рубежом $[18,19,20,21]$. Однако, именно проблема влияния гипервентиляционного синдрома на альтернативные друг другу контингенты обследуемых, исходный кардиологический статус которых скомпрометирован в различной степени, не изучена в надлежащей степени, что доказывает низкий уровень ее научной разработанности.

На основе детализированного теоретического анализа выявленной проблемы были сформулированы следующие «рабочие» научные гипотезы:

- гипервентиляционный синдром является актуальной патогенетической проблемой, как для условно здоровых лиц, так и для пациентов с различными по тяжести формами кардиальной патологии;

- при остром коронарном синдроме (в виде нестабильной стенокардии), сочетанном с сахарным диабетом второго типа, «присоединение» к его клинической картине гипервентиляционного синдрома в значительной степени ухудшает течение и исход заболевания.

С целью разрешения выявленной проблемы, а также доказательств выдвинутых научных гипотез, запланировано и реализовано следующее трехстадийное исследование.

\section{Шель исслеАования}

Изучение соматической значимости гипервентиляционного синдрома для обследуемых с различным исходным кардиальным статусом (на примере условно здоровых лиц молодого возраста, пациентов с хрониче- ской сердечной недостаточностью пожилого возраста, пациентов с нестабильной стенокардией и сопутствующим сахарным диабетом второго типа).

\section{Материалы и метомы}

Исследование реализовано в виде трех последовательных стадий.

Первая стадия исследования была посвящена изучению патологического значения присутствия гипервентиляционного синдрома у условно здоровых лиц молодого возраста.

Вторая стадия исследования была посвящена изучению патологического значения присутствия гипервентиляционного синдрома у пациентов пожилого возраста с хронической сердечной недостаточностью (ХCH).

Третья стадия исследования была посвящена изучению патологического значения присутствия гипервентиляционного синдрома у пациентов с нестабильной стенокардией на фоне сахарного диабета второго типа.

Методологическая платформа настоящей работы включила корректный дизайн исследования, применение логически обоснованных показателей, обоснованных методов их оценки и статистическую проверку всех эмпирических данных и положений выдвинутых научных гипотез. Проведенное исследование соответствовало принципам систематичности, релевантности и непредвзятости.

Проведено комплексное клинико-инструментальное обследование: 1) 68 условно здоровых лиц на примере студентов Медицинского института Тамбовского государственного университета в возрасте 18-23 лет, 2) 48 пациентов, поступивших первично в ГБУз «Тамбовская областная клиническая больница им. В.Д. Бабенко» с диагнозом «Хроническая сердечная недостаточность», в возрасте 55-85 лет, 3) 200 пациентов БУЗ ВО Воронежская областная клиническая больница № 1 с нестабильной стенокардией и сопутствующим сахарным диабетом второго типа (из них - 100 чел. с гипервентиляционным синдромом и 100 чел.- без гипервентиляционного синдрома). Общее количество обследуемых - 316 чел.

Исследовались следующие показатели: 1) гипервентиляционный синдром, 2) стенокардитический болевой синдром, 3) астенический симптомокомплекс, 4) фракция сердечного выброса по УЗИ, 5) исход стенокардии по функциональным классам (ФК). Были применены следующие методы исследования:

- клинические: 1) количественная оценка ГВС по семиотике проявлений, 2) количественная 
оценка болевого синдрома, 3) количественная оценка астенического симптомокомплекса; 4) расчет функционального класса стенокардии;

- инструментальные: 1) капнография, 2) Узи сердца;

- статистические: 1) вариационная статистика определение показателей среднего арифметического (m) и среднего квадратического отклонения ( \pm s) изучаемых показателей в исследуемых группах, 2) оценка распределения случайной величины ( $\boldsymbol{W}$-критерий Шапиро-Уилка), 3) оценка статистической значимости межгрупповых различий (параметрический $\boldsymbol{t}$-критерий Стьюдента для несвязанных выборок), 4) анализ таблиц сопряженности с определением показателей абсолютного риска (AР), прироста абсолютного риска (ПАР) и отношения шансов (ОШ), 5) сравнение наблюдаемых и ожидаемых частот с помощью критерия согласия Пирсона $X^{2}$ с определением его фактического значения и степеней свободы $(\boldsymbol{d} f)$; минимальный принятый уровень статистической значимости результатов $\mathbf{p}<0,05$ [Флетчер Р. и др., 1998; Реброва, 2002; WAME, 2021].

Основное техническое оснащзение: 1) Наймигенский опросник (Nijmegen questionnaire) на предмет гипервентиляционного синдрома, 2) визуальная аналоговая (ВАШ) и цифровая рейтинговая (ЦРШ) шкалы количественной оценки болевого синдрома, 3) Шкала оценки астенического симптомокомплекса Л.Д. Малковой в адаптации Т.Г. Чертовой, 4) Классификация функциональных классов стенокардии Канадского кардиологического общества (1976), 5) аппаратно-программные капнографические комплексы «NICO ${ }_{2}$ («Novametrix Medical Systems Inc.», США) и «CapnoSream 20p» («Oridion medical», Израиль), 6) аппарат УЗИ сердца экспертного класса (УЗИ-сканер) «Vivid E95» («General Electric», США), 7) персональный компьютер с прикладным программным обеспечением StatSoft Statistica 6.0.

О состоятельности выдвинутых в исследовании научных гипотез и эффективности предлагаемых терапевтических подходов свидетельствуют полученные результаты.

\section{Результаты и их обсужление}

Результать первой стадии исследования показали следующее. Было установлено, что у условно здоровых лиц молодого возраста гипервентиляционный синдром имеет клинически ориентированное значение. Так, он обладает высокой частотой встречаемости (78\%), превышающей таковую для нормо- $(20,5 \%)$ и гиповентиляционного (1,5\%) состояния. С высокой долей вероятности он развивается по причине состояния тревожно- сти (26,4\%), последствий учебной нагрузки (22,6\%), чаще всего - при сочетании этих причин (50,9\%).

Как правило, он обладает явным течением, т.е. реализуется в виде одышки (86,7\%), намного реже обладает латентным течением в виде только капнографических проявлений без ее развития (13,2\%). В подавляющем большинстве случаев $(88,7 \%)$ он приводит к негативным субклиническим последствиям в виде периодически возникающих головных болей (39,48\%), астенизации $(34,76 \%)$, тревожности / беспокойства (29,1\%), аэрофагии (45,08\%), пароксизмальной тахикардии (18,12\%), эмоциональной лабильности (14,4\%), диссомнии (10,89\%), чего не имеет или почти не имеет места при его отсутствии; без таковых последствий протекает намного реже (11,3\%). В совокупности эти результаты показали клинически важное значение гипервентиляционного синдрома даже у условно здоровых лиц молодого возраста.

Интерпретация полученных результатов при их обследовании явилась мотивирующим фактором для изучения клинико-патогенетической значимости гипервентиляционного синдрома у более «серьезной» медико-социальной группы - кардиологически скомпрометированных пациентов с ХCH, что было изучено на второй стадии предварительных исследований.

Результаты второй стадии исследования показали следующее. Было установлено, что ГВС у данных пожилых пациентов с ХСН также имеет клинически ориентированное значение. В частности, он также обладает высокой частотой встречаемости $(70,8 \%)$, значительно превышающей таковую для нормовентиляционного $(29,2 \%)$ состояния. Развивается в силу кардиальных или сочетанных причин, т.к. чисто нейрогенные и иные экстракардиальные причины у тематических пациентов на этапе включения в исследование были исключены. Как правило, обладает явным течением, т.е. реализуется в виде базисного симптома - одышки (91,2\%), очень редко обладает латентным течением в виде только капнографических проявлений без развития одышки (8,8\%). В абсолютном большинстве случаев $(97,1 \%)$ ГВС при ХСН приводил к негативным клиническим последствиям в виде «физического чувства внутреннего напряжения» $(94,1 \%)$, «болевого синдрома в грудной клетке» $(23,5 \%)$, нарушений сердечного ритма (20,6\%), «невозможности полноценного вздоха» (88,2\%), астенизации (94,1\%), чувства тревоги и страха (38,2\%), чего не имело или почти не имело места при нормовентиляционном состоянии (некоторое исключение составлял астенический симптомокомплекс, частота развития которого оставалась высокой - на уровне 71\%, но, тем не менее, по сравнению с ГВС она снижалась на 23,1\%). При этом частота встречаемости ГВС без таковых последствий стремилась к минимуму (2,9\%). Полученные результаты показали ис- 
ключительное клиническое значение гипервентиляционного синдрома у пациентов с хронической сердечной недостаточностью. Также необходимо отметить, что его значимость для пожилых пациентов с ХCH значительно превышала таковую для здоровых лиц молодого возраста.

Интерпретация совокупных результатов первой и второй стадий исследования - при изучении как условно здоровых лиц молодого возраста, так и пациентов пожилого возраста с ХCH - явилась серьезным мотивирующим фактором для последующего изучения клинико-терапевтической значимости гипервентиляционного синдрома для одной из наиболее «экстремальных» групп кардиологических пациентов - с наличием нестабильной стенокардии, осложненной сопутствующими хроническими метаболическими нарушениями в виде сахарного диабета второго типа, в составе третьей стадии исследования.

Результатьл третьей стадии исследований показали следующее. Необходимо отметить, что пациенты сравниваемых групп («с ГВС» / «без ГВС») по всем оцениваемым показателям были статистически неразличимы в начальной (непосредственно перед реализацией периода интенсивного лечения) точке наблюдения (параметрический ткритерий Стьюдента, $\boldsymbol{p}>0,05$ для всех декларируемых случаях межсрупповых различий). Однако, в дальнейшем по многим показателям между группами были выявлены статистически значимые различия.

Было установлено, что наличие гипервентиляционного синдрома по сравнению с его отсутствием:

- обладает негативным воздействием на течение основного заболевания в аспекте экспрессии стенокардитического болевого синдрома; это было доказано тем, что: 1) «среднестатистическая» интенсивность болевого синдрома была выше в 2 раза, 2) количество пациентов с некупированным до конца болевым синдромом по истечению первых суток от начала лечения - больше в 2,12 раза, 3) максимальный период времени от начала лечения, в течение которого может проявляться стенокардитический болевой синдром, был продолжительнее в 1,6 раза;

- обладает негативным воздействием на течение основного заболевания в аспекте экспрессии астенического симптомокомплекса; это было доказано тем, что по сравнению с нормовентиляционным состоянием количество пациентов с проявлениями астенизации: 1) в «середине» периода наблюдения было большим в 1,6 раза, 2) по итогам периода наблюдения - большим в 2,75 раз.
- в статистически значимом количестве случаев (а именно - в 38\%) усугубляет развитие сердечной недостаточности за счет способствования снижению фракции сердечного выброса; при этом риск такого последствия является достаточно высоким $\left(\mathrm{AP}_{2 в c^{+}}=73 \%\right.$, ОШ = 4,93 y.e. $)$;

- является изолированной причиной одышки в $14 \%$ случаев, причиной которых не является миокардиальная дисфункция;

- обладает статистически значимым влиянием на распределение исходов основного заболевания по функциональным классам: 1) группу пациентов без ГВС в наибольшей степени характеризуют второй функциональный класс, третий и первый функциональные классы занимают 2-е и 3-е места соответственно; 2) группу пациентов c $Г В C$, напротив, в наибольшей степени характеризует третий функциональный класс, второй и первый функциональные классы занимают 2-е и 3-е места соответственно; 3) снижает количество пациентов с ФК I - в 2,14 раз, с ФК II - в 1,22 раза, количество же пациентов с ФК III, напротив, повышает в 1,4 раза $\left(x^{2}=15,4402, \boldsymbol{d} \boldsymbol{f}=2, \mathrm{p}<0,000443\right)$.

Полученные результаты свидетельствовали о статистически подтвержденном негативном влиянии гипервентиляционного синдрома на течение и исходы нестабильной стенокардии на фоне сахарного диабета второго типа.

\section{Зак^ючение}

Таким образом, выдвинутая научная гипотеза о том, что гипервентиляционный синдром является актуальной патогенетической проблемой, как для условно здоровых лиц, так и для пацчиентов с различными по тяжести формами кардиологической патологии, подтверждается тем, что:

- у условно здоровых лиц молодого возраста он приводит к негативным субклиническим последствиям в виде головных болей, астенизации, тревожности / беспокойства, аэрофагии, диссомнии, эмоциональной лабильности и даже кардиологически ориентированной симптоматики в виде тахикардии,

- у относительно «легких» кардиологических пациентов - с хронической сердечной недостаточностью - он приводит к негативным клиническим последствиям в виде эмоциональной лабильности, соматоформным проявлениям, а также одышки смешанного генеза и нарушениям сердечного ритма,

- у наиболее «тяжелых» кардиологических пациентов - с острым коронарным синдромом в виде нестабильной стенокардии - он приводит к ухудшению течения и исходов заболевания, 
- при этом у всех перечисленных групп обследуемых он обладает достаточно высокой частотой встречаемости и приводит при этом к, как минимум, одному из кардиологических или кардиологически ориентированных последствий (одышке, тахикардии, аритмии, усугублению стенокардитического болевого синдрома), т.е. обладает высоким уровнем соматической значимости.

Выдвинутая научная гипотеза о том, что при остром коронарном синдроме (в виде нестабильной стенокардии), сочетанном с сахарным диабетом второго типа, «присоединение» к его клинической картине гипервентиляционного синдрома в значительной степени ухудшает течение и исход, подтверждается тем, что:
- течение основного заболевания становится хуже потому, что происходит усугубление стенокардитического болевого синдрома, астенического симптомокомплекса и состояния сниженного сердечного выброса,

- исходы основного заболевания становятся хуже потому, что к моменту завершения этапа интенсивной терапии отмечается более низкий уровень толерантности к физической нагрузке, что соответствует преобладанию третьего функционального класса и общему распределению исходов в виде «ФК III > ФК II > ФК I».

Достигнутые результаты позволяют рекомендовать их к дальнейшему учету в практике здравоохранения на уровне клиники внутренних болезней.

\section{ЛИТЕРАТУРА}

1. Абросимов В.Н. 1991. Гипервентиляционный синдром в клинике внутренних болезней: дис. ... д-ра мед. наук. Рязань. 239.

2. Агаджанян Н.А. 2002. Гипокапнические и гиперкапнические состояния. Москва. 35.

3. Александров А.А. и др. 2005 Сахарный диабет и ишемическая болезнь сердца: поиски решения. Сахарный диабет. 1: 34-38.

4. Бахмутова Ю.В. 2009. Диагностика и коррекция гипервентиляционного синдрома у больных сахарным диабетом посредством Б0С-С02-технологии. Международный журнал прикладных и фундаментальных исследований. 6: 36-39.

5. Борзенко С.Я. 2021. Гипервентиляционный синдром (синдром вегетососудистой дистонии). http://moi03.ru/node/42 [электронный ресурс]. 42

6. Дедов И.И. и др. 2013. Эндокринология: национальное руководство. Москва. 752.

7. Малая Л. 2007. Хроническая сердечная недостаточность. Новейший справочник. Москва. 960.

8. Малов Ю.С. 2014. Хроническая сердечная недостаточность (патогенез, клиника, диагностика, лечение). Санкт-Петербург. 320. ISBN: 978-5-299-00629-2.

9. Марченко В.Н. 2021. Коморбидный больной в практике терапевта ИБС и ХОБЛ. https://petrsu.ru/files/user/3324613444dafceb8c73c8ded946eed1/Mapченк0\%20В.Н.\%2 <0Коморбидный\%20больной.pdf (дата обращения: 01.11.2021)62.

10. Митьковская Н.П. 2016. Кардиология и коморбидность. «І Международный конгресс кардиологов и терапевтов: 12-13 мая 2016 г». Минск: $173-177$.

11. Панина М.И. 2003. Влияние гипервентиляции на иммунологические и биохимические показатели крови у здоровых людей. Казанский медицинский журнал. 82 (2): $85-89$.

12. Реброва 0.Ю. 2002. Статистический анализ медицинских данных: применение пакета прикладных программ STATISTICA. Mockва. 312.

13. Стародубов В.И. 2014. Общественное здоровье и здравоохранение: национальное руководство / В.И. Стародубов. Москва. 624. ISBN: 9978-5-9704-29099.

14. Токарева Н.А. 2004. Гипервентиляционный синдром при соматической патологии (на примере бронхиальной астмы, гипертонической болезни) и органном неврозе — особенности течения и лечения: дис. ... канд. мед. наук. Москва. 137.

15. Флетчер Р., Флэтчер С., Вагнер Э. 1998. Клиническая эпидемиология: основы доказательной медицины. Москва. 352 с.

16. Чазов Е.И. 2000. Проблема лечения больных ишемической болезнью сердца. Терапевтический архив. 9: 59.

17. Шляхто Е.В. и др. 2021. Кардиология: национальное руководство. Москва. 800. ISBN978-5-9704-6092-4.

18. Bausewein C. et al. 2007. Measurement of breathlessness in advanced disease: a systematic review. Respiratory Medicine. 101: 399-410.

19. Da Costa J.M. 1871. On Irritable Heart: a Clinical Study of a Form of Functional Cardiac Disorder and its Consequences. The American journal of the medical sciences. 121 (1): 252.

20. Stoeckel M.C et al. 2017. Dyspnea catastrophizing and neural activations during the anticipation and perception of dyspnea. Psychophysiology. 55 (2): 1-10. D0l: 10.1111/psyp.13004.

21. World Association of Medical Editors (WAME). 2021. http://www.wame.org (дата обращения: 01.11.2021).20-22

○ Воронин Никита Игоревич ( nikita-tmb@yandex.ru ), Кузнецов Сергей Иванович ( nikita-tmb@yandex.ru ),

$$
\text { Кузнецов Дмитрий Сергеевич. }
$$

Журнал «Современная наука: актуальные проблемы теории и практики» 